\title{
La diffusion d'un changement radical : un processus de redéfinition et de restructuration de l'organisation
}

\section{Christiane Demers}

\section{(2) OpenEdition}

\section{Journals}

Édition électronique

URL : http://journals.openedition.org/communicationorganisation/1621

DOI : 10.4000/communicationorganisation. 1621

ISSN : 1775-3546

Éditeur

Presses universitaires de Bordeaux

Édition imprimée

Date de publication : 1 mai 1993

ISSN : 1168-5549

Référence électronique

Christiane Demers, "La diffusion d'un changement radical : un processus de redéfinition et de restructuration de l'organisation », Communication et organisation [En ligne], 3| 1993, mis en ligne le 17 avril 2012, consulté le 10 décembre 2020. URL : http://journals.openedition.org/ communicationorganisation/1621 ; DOI : https://doi.org/10.4000/communicationorganisation.1621

Ce document a été généré automatiquement le 10 décembre 2020.

(C) Presses universitaires de Bordeaux 


\title{
La diffusion d'un changement radical : un processus de redéfinition et de restructuration de l'organisation
}

\author{
Christiane Demers
}

1 Il est généralement admis que le succès d'un changement radical dépend de l'habileté des dirigeants à guider une réorientation cognitive (Bartunek, 1984; Hedberg, 1974), une révolution culturelle (Firsirotu, 1984), à imprégner l'organisation d'une nouvelle vision stratégique (Mintzberg \& Waters, 1983). En effet, un changement est qualifié de radical lorsqu'il entraîne des changements importants, voire des ruptures, tant au niveau de la stratégie, que de la structure et de la culture de l'organisation (Allaire \& Firsirotu, 1985 ; Hafsi \& Demers, 1989 ; Tushman \& Romanelli, 1985). Le changement radical est donc un changement de deuxième ordre (Ginsberg, 1988; Ramaprasad, 1982), c'est-à-dire un changement global qui remet en cause le cadre de référence dominant (Nadler \& Tushman, 1989).

Or, les périodes de discontinuité, selon Hedberg \& Jönsson (1977), accentueraient les divergences entre points de vue. Dans ces conditions, la diffusion d'une nouvelle stratégie serait donc un défi majeur pour les gestionnaires; surtout dans les organisations complexes où la diversité des perspectives (Hafsi, 1985; Simon, 1976) rend difficile le développement d'une vision partagée.

Les changements radicaux étant de plus en plus fréquents (Allaire \& Firsirotu, 1985 ; Hafsi \& Demers, 1989), l'étude de la diffusion stratégique apparaît donc particulièrement pertinente. Il est, en effet, plausible de croire que la compréhension qu'ont les membres d'une nouvelle stratégie, ainsi que le support qu'ils lui accordent, dépendent, entre autres, du processus grâce auquel ils en prennent connaissance et lui donnent un sens. Dans cette perspective, le processus de diffusion stratégique doit être conçu non seulement comme la simple transmission d'information à des récepteurs passifs, il est 
également un processus interactif de création de sens, c'est-à-dire un processus d'interprétation.

4 À ce jour, notre connaissance du processus de diffusion stratégique dans les situations de changement radical est très limitée. Les quelques études empiriques sur le changement radical (Anderson, 1986; Bibeault, 1982 ; Firsirotu, 1984) ont plutôt mis l'accent sur les actions de la haute direction et ne décrivent qu'en termes unidirectionnels la diffusion du changement. Elles ne tiennent donc pas compte explicitement de l'interprétation et de l'action des autres membres de l'organisation. Une des rares exceptions est l'étude de Bartunek (1984) sur le changement de mission et la restructuration d'une communauté religieuse. Cependant, ce cas est particulier du fait qu'il traite d'un changement émergent plutôt que planifié : un changement qui, dès le départ, est très décentralisé plutôt que contrôlé par la haute direction. En fait, ces études représentent deux courants différents de la littérature en stratégie qui suggèrent deux conceptions du processus de diffusion que nous allons présenter dans la prochaine section.

Dans le but de mieux comprendre le processus de diffusion stratégique, nous avons réalisé une étude de terrain intensive à Hydro-Québec, le monopole de production et de distribution d'électricité au Québec, qui vivait un changement radical planifié par la haute direction (Demers, 1990). Il s'agit d'une étude longitudinale basée sur l'approche interprétative, donc centrée sur l'acteur, qui visait à développer un modèle théorique de la diffusion stratégique fondé sur les données empiriques, dans le sens de la "grounded theory " proposée par Glaser \& Strauss (1976). Les données couvrent la période allant de 1982 à 1988 et ont été recueillies sur une période de trois ans, du début de 1986 à la fin de 1988. Elles proviennent de diverses sources, entre autres, de l'étude de documents, d'échanges informels durant une période d'observation participante et, principalement, d'entrevues non structurées.

6 Prenant appui sur cette étude et sur la littérature, cet article propose un modèle du processus de diffusion stratégique. Ce modèle intègre processus de transmission d'information et processus d'interprétation et tient compte de l'interaction entre la vision de la haute direction et les interprétations des autres membres de l'organisation.

\section{Le processus de diffusion stratégique}

Il existe, dans la littérature en stratégie, différents modèles du processus stratégique qui peuvent être situés sur un continuum allant du modèle rationnel au modèle émergent (Bourgeois \& Brodwin, 1984). Le modèle dominant est le modèle rationnel et linéaire, où la formulation de la stratégie, activité exclusive de la haute direction, précède et détermine la mise en œuvre, activité où les autres membres de l'organisation sont avant tout des exécutants (Andrews, 1987). Le modèle émergent, plus récent, conçoit la formulation et la mise en œuvre de la stratégie comme des processus simultanés et inter reliés auxquels participe toute l'organisation, la vision émanant de l'action autant que la suscitant (Mintzberg \& Waters, 1985). De ces modèles découlent deux conceptions fort différentes du processus de diffusion stratégique que nous appelons le modèle informationnel et le modèle interprétatif (voir tableau 1). 


\begin{tabular}{|c|c|c|}
\hline & $\begin{array}{l}\text { INFORMA- } \\
\text { TIONNEL }\end{array}$ & INTERPRETATIF \\
\hline $\begin{array}{l}\text { FONTION DU } \\
\text { MESSAGE }\end{array}$ & informative & $\begin{array}{l}\text { informative et } \\
\text { symbolique }\end{array}$ \\
\hline $\begin{array}{l}\text { VALEUR DU } \\
\text { MESSAGE }\end{array}$ & $\begin{array}{l}\text { factuelle/ } \\
\text { évidence }\end{array}$ & polysémique \\
\hline OBJECTIF & $\begin{array}{l}\text { reprodution/ } \\
\text { acquiescement }\end{array}$ & $\begin{array}{l}\text { traduction/ } \\
\text { appropriation }\end{array}$ \\
\hline RESULTAT & $\begin{array}{c}\text { info } \mathrm{T}_{0}=\text { info } \mathrm{T}_{1} \\
\text { pour a pour } \mathrm{b}\end{array}$ & $\begin{array}{c}\text { info } T_{1} \geq \text { info } T_{0} \\
\text { pour a pour a }\end{array}$ \\
\hline
\end{tabular}

\section{Le modèle informationnel}

Dans le contexte du modèle rationnel, la diffusion de la stratégie est une des phases du processus d'implantation et est conçue essentiellement comme un processus de transmission d'information à tendance unidirectionnelle. Cette conception se rapproche du modèle de communication traditionnel et de la perspective véhiculée par les études sur la diffusion des innovations (Rogers \& Shoemaker, 1971). Dans le cas des organisations, celles-ci traitent de la propagation d'innovations, tel un nouveau produit, une nouvelle technique, et ont été faites principalement par des chercheurs qui s'intéressent au marketing et à l'innovation technologique ou managériale. Ces auteurs adoptent une perspective qui met l'accent sur l'aspect transmission d'information, c'està-dire le mouvement de l'innovation dans l'espace organisationnel. Les aspects structurels et temporels de la diffusion sont mis en évidence.

Cette perspective laisse de côté les phénomènes de sens. La création d'information étant considérée l'apanage exclusif de la haute direction, il en résulte, comme le souligne Nonaka (1988), que le but premier de la diffusion est la réduction de variété, c'est-à-dire la reproduction la plus fidèle possible de la vision des dirigeants. L'organisation étant conçue comme un monolithe où un cadre de référence domine, tout message est perçu comme ayant une seule signification possible. L'existence d'interprétations différentes est traitée comme un problème résultant d'ignorance (manque d'information), de bruit (mauvaise qualité de l'information) ou de mauvaise volonté (résistance face au changement) qu'une mise en œuvre réussie peut surmonter (Hrebeniak \& Joyce, 1984). 


\section{Le modèle interprétatif}

10 Pour le modèle émergent, le processus de diffusion est un processus de création de sens auquel peut participer toute l'organisation et, en ce sens, il découle d'une perspective interprétative (Chaffee, 1985 ; Isabella ; 1990). Ce modèle suggère l'existence, à l'intérieur de l'organisation, de groupes aux cadres de référence différents qui réinterprètent la réalité organisationnelle de façon à y intégrer leurs activités et qui ainsi stimulent le renouvellement organisationnel. Dans cette perspective, le processus de diffusion est un processus de traduction et de redéfinition de la nouvelle stratégie. Les problèmes de diffusion propres à ce modèle qui sont suggérés dans la littérature sont, entre autres, la cohérence interne (Burgelman, 1983; Mintzberg \& Waters, 1985 ; Nonaka, 1988) et la légitimation (Pettigrew, 1985).

11 Toutefois, les auteurs qui s'intéressent spécifiquement au processus d'interprétation dans les organisations, ont, jusqu'à présent, généralement mis l'emphase sur l'interface organisation-environnement (Daft \& Weick, 1984 ; Daft \& Huber, 1987) et considéré avant tout l'impact de la haute direction sur le processus d'interprétation (Isabella, 1990; Neilsen \& Hayagreeva Rao, 1987). Une des conséquences de cette orientation est d'avoir étudié le processus d'interprétation organisationnel comme un processus désincarné: une activité avant tout cognitive, plutôt qu'une activité sociale ancrée dans un univers structurel et temporel. C'est pourquoi certains auteurs, dont Chaffee (1985), Cummings (1983) et Krone et al (1987) préconisent une analyse intégrée des modèles informationnel et interprétatif.

\section{La diffusion stratégique : transmission et interprétation}

12 L'objectif de ce travail est donc de combiner ces deux perspectives, c'est-à-dire de considérer la diffusion à la fois en tant que processus de transmission d'information et processus d'interprétation. La dimension transmission d'information met l'accent sur le mouvement de la nouvelle stratégie dans l'espace organisationnel. Une organisation complexe étant un espace structuré, la circulation de l'information y est contrainte par des règles et des réseaux formels et informels dont il faut tenir compte si l'on veut comprendre comment se diffuse la stratégie. La dimension interprétation, quant à elle, met en relief la création de sens, l'évolution des cadres de référence des différents groupes. En effet, à cause des différences de perspectives, l'information se transforme et change de signification lorsqu'elle circule dans l'organisation.

Dans les pages qui suivent, nous allons proposer un modèle intégré du processus de diffusion qui permet de tenir compte à la fois de la perspective de la direction et de celles des autres membres de l'organisation dans une situation de changement radical. Nous examinerons ensuite les avenues de recherche qui découlent de ce modèle. 


\section{La diffusion : un processus de redéfinition et de restructuration}

14 Le changement radical, par définition, entraîne des changements importants au niveau de la stratégie et de la structure de l'entreprise. Il s'agit d'une période durant laquelle la mission de l'organisation est transformée, alors même que les réseaux de diffusion sont détruits et doivent être reconstruits. C'est donc dire que, dans une situation de changement radical, le processus d'interprétation est un processus de redéfinition de la réalité organisationnelle, de transformation du cadre dominant, alors que le processus de transmission de la nouvelle stratégie est un processus de reconstruction des réseaux, de restructuration de l'entreprise.

Or, certains auteurs, entre autres Ranson \& Hinings (1980) et Bartunek (1984), qui s'inscrivent dans une perspective structurationniste (Giddens, 1984), soutiennent que la restructuration d'une organisation et la redéfinition de la réalité organisationnelle sont intimement liées. En effet, les interactions entre les membres de l'organisation recréent, à la fois, le cadre de référence organisationnel et les réseaux structurels. Le processus de diffusion peut donc être conçu comme l'interaction entre les processus de redéfinition et de restructuration de l'organisation ; mais comment ce processus de diffusion s'articule-til ?

Le modèle que nous proposons conçoit la diffusion d'un changement radical initié par la haute direction comme l'interaction entre deux dynamiques qui s'articulent, respectivement, autour de la redéfinition de la mission (ou l'articulation de la stratégie) et de la redéfinition de la gestion (ou la mise en œuvre au sens large). La première est une dynamique horizontale: une négociation entre les différentes unités de l'organisation, tandis que la deuxième est une dynamique verticale: une chaîne d'échanges entre supérieur et subordonné. Comme nous allons le voir chacun d'eux présente des caractéristiques particulières (voir le tableau 2).

\section{La redéfinition de la mission : une dynamique horizontale}

17 La redéfinition de la mission d'une organisation complexe porte, en grande partie, sur la spécification des activités de chacune des unités de l'entreprise et du degré d'intégration nécessaire entre elles. Le focus de cette conversation est, en fait, la délimitation de l'espace organisationnel, des territoires qui le composent, de ses frontières et de son ouverture face à l'environnement.

Nous qualifions ce processus de dynamique horizontale parce qu'il restructure latéralement l'organisation, c'est essentiellement une négociation entre les différentes unités de l'organisation (domaines d'activités, groupes fonctionnels), qui vise à spécifier ce que va faire l'organisation dans le futur et la place de chaque unité dans cette nouvelle réalité. Ce processus de diffusion recrée le réseau et les règles d'interaction entre les différentes parties de l'organisation.

Par exemple, au début des années 80 , suite à une baisse de la demande, Hydro-Québec, le monopole québécois de production et de distribution d'électricité au Québec, décide de se 
transformer d'« un constructeur de barrages", réputé internationalement pour son expertise dans la réalisation de mégaprojets, en "vendeur d'électricité », conscient des coûts et tourné vers la clientèle. Ce «virage commercial» implique des changements importants dans le rôle qui est dévolu à chaque secteur d'activité. Alors qu'auparavant le groupe Équipement, responsable de la construction des nouvelles installations hydroélectriques, était le moteur de l'entreprise, c'est maintenant le groupe Exploitation, chargé des opérations quotidiennes et des liens avec la clientèle, qui prend ce rôle. De plus, ce changement amène une plus grande interdépendance entre les groupes d'activités qui ne doivent plus se fier uniquement à l'évolution «naturelle» de la demande, mais également aux attentes des divers clients, pour planifier leurs activités.

C'est dans le cadre de la redéfinition de la mission que les perspectives propres aux différents domaines d'activités se confrontent. Cette redéfinition est axée sur la construction d'un nouveau contexte de signification commun: un nouveau langage, de nouveaux concepts de ce qu'est l'organisation, de ce qu'elle va devenir. Par ailleurs, si les divergences entre les domaines d'activités ne sont pas transcendées, elles peuvent amener à la fragmentation stratégique. Ce processus articule dans le temps la tension différenciation-intégration décrite par Lawrence \& Lorsch (1969).

Les participants à cette négociation sont généralement de niveaux hiérarchiques équivalents et ont des préoccupations de même nature. Ils ont le même niveau de discours, ce qui facilite la discussion. Leur attention va aux problèmes de direction générale. De plus, si l'esprit corporatif est développé, les membres de la haute direction forment un groupe d'appartenance : ils s'identifient plus au groupe de direction qu'à leur unité respective.

Les participants à la discussion sont, en principe, des pairs. Puisque les relations d'autorité ne sont pas mises en évidence dans ce contexte, l'interaction tend à être plus informelle, et c'est l'influence qu'a une personne sur ses partenaires (plutôt que l'autorité formelle) qui détermine le pouvoir qu'elle a sur les décisions qui sont prises. Dans ce contexte, il est primordial de parler un langage que les autres comprennent. Cela suppose que les dirigeants soient capables de traduire en langage corporatif le discours de leur groupe respectif et vice-versa. Les membres de la direction, lors d'un changement radical, doivent donc arriver à intégrer deux discours.

\section{Le cycle d'exclusion ou l'importance de la représentativité}

Si on se place du point de vue de l'ensemble des membres de l'organisation, le processus de diffusion horizontal est caractérisé par le fait que les membres des groupes n'y participent pas directement. Les responsables des groupes y parlent, implicitement, au nom de leurs membres. Dans ce contexte, la communication entre la haute direction et la base est donc médiatisée. Dans cette dynamique, l'interprétation du changement qu'ont les membres du groupe dépend de la crédibilité de leur responsable, c'est-à-dire de leur confiance en ses capacités à les représenter adéquatement. Un responsable de groupe pour être perçu comme un représentant efficace doit être à la fois un défenseur des intérêts du groupe et un membre influent de l'équipe de direction. transformée. Cela soulève pour les groupes dont le dirigeant est changé, la question de 
représentativité. En effet, dans une situation de changement radical, le remplacement du dirigeant est un signal puissant pour le groupe concerné qu'il est sujet à une sérieuse remise en cause. Si le nouveau responsable est perçu comme étant uniquement l'émissaire de la nouvelle direction, ce groupe entre dans ce que nous appelons un cycle d'exclusion (voir tableau 3). Les membres du groupe ont le sentiment d'être exclus du processus de diffusion horizontal, de ne pas participer à la transformation de leur territoire, mais plutôt de la subir. Puisque personne ne parle en leur nom, ne défend leurs intérêts, ils ne se sentent pas partie prenante de cette négociation.

Il s'ensuit que la nouvelle stratégie est, à leurs yeux, incomplète, parce qu'elle ne tient pas compte de la réalité du groupe. Pour les membres des groupes qui se sentent exclus, la nouvelle vision est donc dénuée de sens profond. À Hydro-Québec, par exemple, c'est ce qui s'est passé, dans un premier temps, avec le groupe Équipement où la majorité des membres avaient le sentiment d'avoir été trahis par l'entreprise. Les commentaires qui suivent reflètent bien cette perception :

«À Équipement, on a été les dindons de la farce; il y a eu de la désillusion. Tant qu'on nous demandait de construire des gros projets - même on nous demandait de les devancer - là on faisait de grandes réalisations. Quand il y a eu de la décroissance, on était encombrants. » (chef de service)

«Je pense que l'importance du groupe Équipement a diminué... On a été vu comme les gros méchants. Tous les problèmes étaient causés par nous : on planifiait mal, on calculait mal... » (un ingénieur)

Ce genre de situation conduit à une incompréhension de la nouvelle stratégie parce que les gens des groupes exclus ne voient pas clairement le rôle qu'ils doivent jouer :

"J'ai l'impression qu'il y en a beaucoup qui ne comprennent pas où s'en va le groupe Équipement et les gens aimeraient le savoir... » (chef de chantier)

«On avait l'impression qu'ils avaient quelque chose derrière la tête. On disait qu'on voulait un groupe Équipement fort, mais ce qu'on voyait c'est qu'Équipement en perdait des bouts... Pour moi, ce n'est pas clair ce qu'ils voulaient faire. S'ils voulaient éliminer Équipement, ils n'ont pas réussi ; s'ils ne voulaient pas le faire, ils l'ont pas mal amoché. » (chef de travaux)

La préoccupation principale des groupes exclus est de réintégrer le processus de redéfinition des territoires. Tant que ce n'est pas fait, l'écart entre leur vision des choses et celle de la haute direction va s'agrandissant. Cette situation risque de se poursuivre tant que le responsable du groupe n'a pas acquis de crédibilité en tant que représentant. Souvent, seul son remplacement peut rétablir la situation. Or, cette situation entraîne des problèmes au niveau de la mise en œuvre et, donc, des problèmes au niveau de la redéfinition de la gestion sur lesquels nous reviendrons plus tard.

Jusqu'à présent, nous avons traité le processus de diffusion horizontal qui sous-tend la redéfinition de la mission, comme si seule la haute direction y participait activement. C'est parce que, dans la majorité des changements radicaux, les hauts dirigeants y jouent le rôle le plus important, ce sont eux qui initient et contrôlent le changement. Donc, même lorsque le processus se déroule également diagonalement, par le biais de comités ad hoc et de groupes de réflexion, ces structures parallèles sont perçues, dans les groupes qui se sentent exclus, comme des moyens de manipulation où les gens n'ont pas le choix, où il faut dire ce que la direction souhaite entendre. Ces comités perdent ainsi leur légitimité en tant qu'outil de représentation.

29 Par ailleurs, les groupes qui se sentent inclus dans le processus de redéfinition de la mission, s'identifient à la nouvelle vision de l'organisation. Ils sont prêts à s'engager dans 
la réalisation de ce changement. Toutefois, comme nous allons le voir, la force et la durée de leur engagement dépendra du fonctionnement du processus de redéfinition de la gestion dont nous présenterons les caractéristiques dans la section suivante.

\section{La redéfinition de la gestion : une dynamique verticale}

La redéfinition de la gestion s'articule, entre autres, autour de questions telles : le partage des tâches, la spécification des responsabilités, la marge de manœuvre qui est accordée à chacun et, particulièrement, la gestion du changement. Son focus est donc la mise en œuvre vue au sens large. Il s'agit ici de définir quel genre d'organisation on veut bâtir et le rôle que chaque individu pourra jouer dans le nouvel ordre des choses.

L'unité de base de ce processus de diffusion est l'échange entre supérieur et subordonné ce qui nous amène à la qualifier de dynamique verticale. C'est la chaîne d'interactions entre supérieur et subordonné qui reconstruit la structure hiérarchique. C'est ce processus de diffusion qui est le plus évident lors d'un changement initié par la haute direction, parce qu'il touche directement tous les membres de l'organisation.

En effet, les changements radicaux visent à transformer non seulement la relation de l'organisation à son environnement et sa mission, mais également son fonctionnement interne, ses valeurs, sa culture. Pour reprendre l'exemple d'Hydro-Québec, l'équipe de direction voulait que la société d'État, « tout en demeurant une société de service public... ressemble de plus en plus à une entreprise commerciale». Pour les dirigeants, cela voulait dire, entre autres, que cette entreprise, centralisée, axée sur l'expertise technique, tournée vers l'interne, se décentralise, mette l'accent sur le contrôle des coûts et s'ouvre à l'externe. Toutes ces interventions nécessitent un changement de mentalité important qui se concrétise dans la gestion du changement lui-même, c'est-à-dire dans sa mise en œuvre.

La redéfinition de la gestion est axée sur la construction du corps social; il s'agit, pour reprendre l'expression de Selznick (1957: 90), de transformer « a neutral body of men into a committed polity ». Ici, le défi tel que décrit par Barnard (1968: 233-234) est d'assurer la cohésion de l'ensemble jusque dans les décisions opérationnelles et de maintenir la « vitalité de l'action », la volonté de l'effort. L'objectif de ce processus est de permettre à tous de s'approprier les objectifs, tout en évitant la dérive et la dispersion stratégiques. La tension inhérente en est une entre autonomie et contrôle qui se traite souvent en termes de centralisation/décentralisation dans la littérature en théorie des organisations (Mintzberg, 1979).

C'est dans la pratique quotidienne, dans le cadre de la relation directe entre supérieur et subordonné, que se construisent les nouvelles façons de faire. Bien que, dans ce processus, les participants soient du même domaine d'activités et donc partagent la même perspective, les différences en termes hiérarchiques font qu'ils ont des niveaux de discours différents. Le responsable du groupe fait partie de la haute direction et tend à développer une vue d'ensemble de l'organisation, tandis que les cadres intermédiaires et de premier niveau qui sont des courroies de transmission entre le sommet et la base ont un point de vue plus opérationnel. Le décalage implicite dans l'interaction en chaîne propre au processus vertical, s'il devient trop important, peut entraîner la stratification de l'entreprise. Ce décalage favorise la perception de contradictions dans la communication supérieur-subordonné (Mc Daniel Johnson, 1977) : dans le temps et entre 
ce qui est dit et ce qui est fait. La capacité du supérieur à adapter son niveau de discours au contexte du subordonné semble donc nécessaire pour permettre à celui-ci de comprendre ce qui se passe, d'en saisir la cohérence.

Tableau 2 - La diffusion stratégique

\begin{tabular}{|c|c|c|}
\hline & $\begin{array}{l}\text { Processus } \\
\text { horizontal }\end{array}$ & $\begin{array}{c}\text { Processus } \\
\text { vertical }\end{array}$ \\
\hline Thème privilégié & $\begin{array}{l}\text { Redéfinition } \\
\text { des territoires }\end{array}$ & $\begin{array}{l}\text { Redéfinition } \\
\text { de la gestion }\end{array}$ \\
\hline $\begin{array}{l}\text { Type de } \\
\text { processus }\end{array}$ & $\begin{array}{c}\text { Négociation entre } \\
\text { les différents } \\
\text { groupes } \\
\text { d'activités }\end{array}$ & $\begin{array}{l}\text { Echange entre } \\
\text { supérieur et } \\
\text { subordonnée }\end{array}$ \\
\hline Type de relation & $\begin{array}{l}\text { Relation } \\
\text { médiatisée basée } \\
\text { sur la crédibilité }\end{array}$ & $\begin{array}{l}\text { Relation directe } \\
\text { permettant une } \\
\text { certaine } \\
\text { réciprocité }\end{array}$ \\
\hline Facteur critique & Représentativité & $\begin{array}{l}\text { Appropriation } \\
\text { des objectifs }\end{array}$ \\
\hline $\begin{array}{l}\text { Dynamique } \\
\text { temporelle }\end{array}$ & $\begin{array}{l}\text { Pertinence } \\
\text { immédiate }\end{array}$ & $\begin{array}{c}\text { Constance dans le } \\
\text { temps }\end{array}$ \\
\hline
\end{tabular}

\section{Le cycle de désengagement ou l'importance de l'appropriation des objectifs}

Il va sans dire que, pour les groupes qui se sentent exclus du processus de redéfinition de la mission, l'appropriation des objectifs est impossible. En effet, seuls les changements qui peuvent être imposés sont réalisés. Dans un sens, les groupes exclus tentent d'utiliser la mise en œuvre pour forcer une redéfinition des territoires. Autrement dit, le processus de redéfinition de la gestion peut devenir un processus de résistance qui, ultimement, mène à un apprentissage des moyens pour miner la vision de la haute direction dans la pratique.

Cependant, même pour les groupes qui se sentent inclus dans la redéfinition de la mission, donc qui sont d'accord avec le changement proposé, l'appropriation des objectifs peut être un problème. Dans une situation de changement radical, la complexité du changement entrepris et le temps qu'il exige rend la cohérence difficile à maintenir. L'existence d'une forme de réciprocité dans la communication supérieur-subordonné peut réduire l'effet du décalage en permettant l'engagement actif dans la mise en œuvre du changement. En effet, c'est dans le contexte de l'échange entre supérieur et subordonné que se fait l'appropriation de la nouvelle stratégie (Westley, 1990). Il ne s'agit pas ici tant de participation formelle à la prise de décision, mais plutôt d'une ouverture de la part du supérieur à discuter de la stratégie et à permettre l'expérimentation au 
niveau de la mise en œuvre, c'est-à-dire la possibilité pour le subordonné de «traduire » la stratégie en fonction de son contexte.

L'engagement des subordonnés semble être lié à leur capacité d'acquérir le pouvoir nécessaire pour agir (Moss Kanter, 1983). Si une discussion réelle des objectifs est impossible, le subordonné ne peut se les approprier et éprouve de la difficulté au niveau de la mise en œuvre. L'interaction se faisant dans le cadre de relations d'autorité formelles, le supérieur peut imposer à son subordonné des objectifs que ce dernier considère irréalisables.

De plus, une relation entre supérieur et subordonné où l'apport de ce dernier n'est pas sollicité fait en sorte que les problèmes de mise en œuvre ne remontent pas la ligne hiérarchique et continuent de s'accroître. Comme la structure verticale est constituée d'une chaîne pyramidale de relations entre supérieur et subordonné, un blocage à un niveau se répercute jusqu'au bas de la ligne hiérarchique et son impact s'amplifie par effet de cascades. Ce blocage affecte aussi le sommet parce qu'il empêche la diffusion ascendante. Les goulots d'étranglement ainsi créés résultent en une mise en œuvre axée sur les résultats à court terme, les seuls qui peuvent facilement être contrôlés.

Une absence de réciprocité dans la relation supérieur-subordonné entraîne les subordonnés dans ce que nous appelons un cycle de désengagement (voir tableau 3). Celui-ci conduit à un apprentissage dysfonctionnel: les gens dans la structure hiérarchique apprennent à " gérer la façade ». Ils deviennent apathiques et se protègent en sauvant les apparences.

À Hydro-Québec, les cadres des régions qui étaient pourtant d'accord au départ avec le changement, ont l'impression que la direction ne tient pas suffisamment compte de ce qu'ils perçoivent être la réalité du terrain. Ils voient, dans la pratique, des incohérences entre les objectifs (par exemple, service à la clientèle et restrictions budgétaires) qui leur font questionner les intentions des dirigeants, donc qui minent la légitimité de la stratégie corporative. Ils ne se sentent pas écoutés et vivent la diffusion verticale comme unidirectionnelle. D'où leur impression que la haute direction est coupée de la réalité du terrain. C'est pourquoi, d'abord, les cadres de premier niveau et, ensuite, les cadres de deuxième niveau, s'associent et nomment un porte-parole pour aller les représenter directement auprès de la haute direction. Le regroupement signifie que le problème est systémique et non pas uniquement interpersonnel; c'est une manifestation de la désintégration du processus de diffusion vertical ${ }^{1}$, avec tout ce que cela comporte comme difficultés de légitimation du changement.

41 Comme le changement est dirigé d'en haut, en l'absence d'information pertinente venant du terrain, cette situation résulte en un écart grandissant entre la stratégie formulée et la stratégie réalisée et, éventuellement, à une remise en cause de la crédibilité du responsable du groupe, comme représentant, et de la vision elle-même.

En effet, les processus de diffusion horizontal et vertical ne sont pas indépendants. D'une part, redéfinition de la mission et redéfinition de la gestion sont toujours étroitement couplés. Par exemple, les gestes posés dans la pratique quotidienne modifient toujours un peu la stratégie, donc redéfinissent partiellement les orientations et le territoire organisationnel. D'autre part, ce sont les mêmes personnes qui font partie des deux processus. Les représentants des groupes d'activités dans le processus de redéfinition de la mission occupent simultanément une place dans la structure hiérarchique et, par le fait 
même, dans le processus de redéfinition de la gestion. Donc, tant en termes d'évolution du contenu que de structuration, ces deux processus forment une matrice d'interaction.

\section{L'interaction entre les deux processus}

Lorsque les processus de diffusion horizontal et vertical fonctionnent harmonieusement, la redéfinition de la mission et la redéfinition de la gestion se renforcent mutuellement, ce que nous appelons le cycle d'intégration (voir tableau 3). La participation de tous les groupes à la redéfinition des orientations enrichit la vision, puisqu'elle amène une synthèse des différentes perspectives qui tient compte, dans une certaine mesure, de la réalité de chacun. Il est alors possible de parler d'une vision commune qui nourrit, pour ainsi dire, la mise en œuvre. Si, à son tour, la redéfinition de la gestion favorise l'appropriation des objectifs, donc l'engagement dans leur réalisation, l'organisation est dans un cycle d'apprentissage qui renforce, à son tour, le processus de définition de la mission. Dans ce contexte, les processus de diffusion horizontal et vertical sont bien intégrés et les liens verticaux et horizontaux se renforcent mutuellement.

Par ailleurs, les logiques d'exclusion et de désengagement engendrent des tensions entre les groupes d'activités et entre les niveaux hiérarchiques qui peuvent mener, ultimement, à la transformation de l'entreprise en une arène politique. Dans un tel cas, l'apprentissage organisationnel devient très difficile ; l'action importante n'est plus celle qui se passe sur le terrain, mais celle qui se passe à l'interne autour de la restructuration des réseaux. Ces deux processus ont donc l'un sur l'autre un effet amplificateur, soit positif, soit négatif. L'analyse des "cercles vicieux" de diffusion suggère certaines difficultés liées à l'interaction entre les deux processus que nous allons maintenant présenter.

Tableau 3 - Modèle dynamique de la diffusion

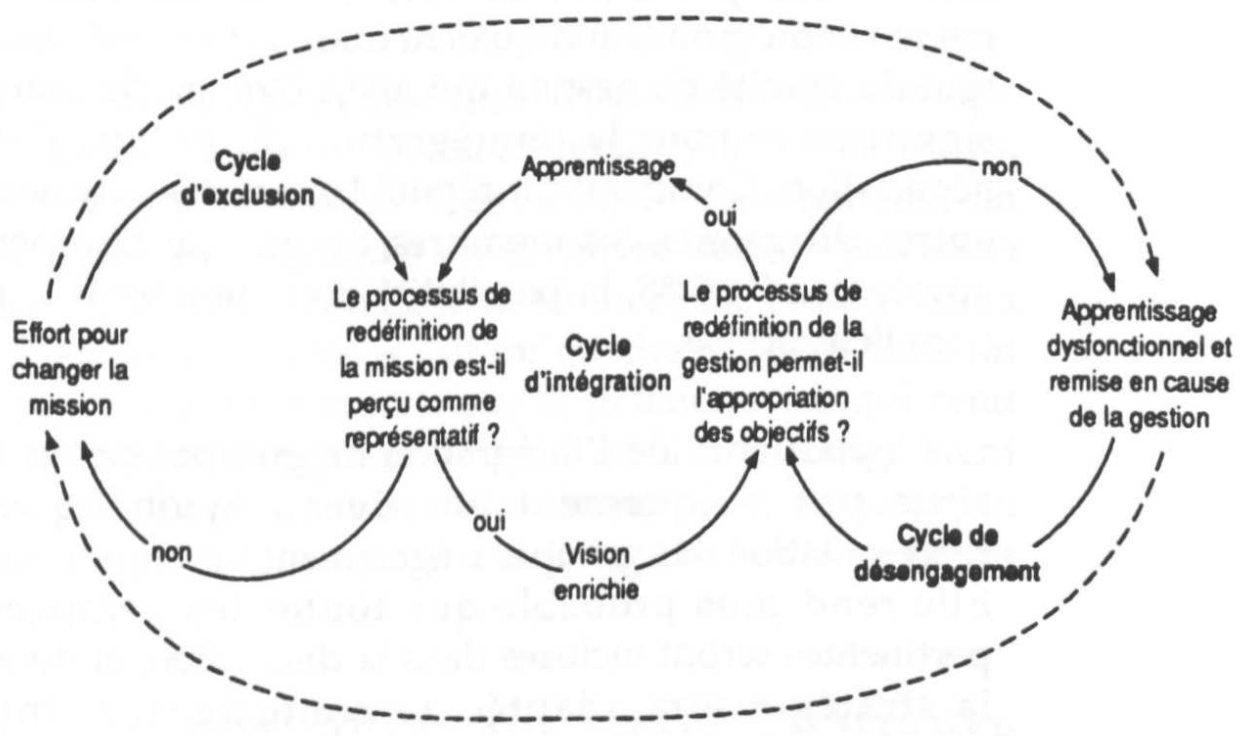




\section{Les arbitrages entre les interprétations différentes} une situation de changement radical, les nouveaux objectifs sont en rupture avec la tradition et l'appropriation des objectifs par la hiérarchie devient donc une question très délicate. À cet égard aussi, la perspective de la direction et celle des autres niveaux hiérarchiques sont potentiellement conflictuelles. Les dirigeants veulent s'assurer que ce qui est réalisé colle le plus possible avec les orientations qui ont été définies, tandis que les gens sur le terrain, même s'ils sont d'accord avec celles-ci, veulent avoir le plus de latitude pour établir les priorités de la façon qui leur semble la plus cohérente et la plus profitable dans la pratique.

51 Donc, le dialogue entre supérieurs et subordonnés qui rend la mise en œuvre possible modifie, en même temps, la stratégie. Il peut aussi bien renforcer que remettre en cause le consensus auquel on est arrivé dans le processus horizontal de définition de la mission. Donc, l'interrelation entre les deux processus fait qu'un changement dans un des processus a un impact sur le deuxième, ce qui rend d'autant plus complexe la gestion d'un changement radical où l'on intervient simultanément, et de façon importante, au niveau de la mission et du fonctionnement interne. 


\section{Les tensions dues aux caractéristiques temporelles des deux processus}

52 Certaines caractéristiques temporelles des deux processus de diffusion créent des tensions supplémentaires dues à leur interaction. Le processus de définition des territoires étant essentiellement une négociation entre les différentes parties de l'organisation, l'état de la situation au moment de la discussion a une grande importance pour le déroulement du débat. Les événements ponctuels qui influent différemment sur chacun des participants menacent le consensus. Dans ce contexte, la priorité va à la pertinence de la solution, compte tenu du contexte immédiat.

53 En conséquence, dans un environnement imprévisible et changeant, le consensus ne peut être atteint qu'à court terme et doit être continuellement révisé. De plus, on peut penser que, plus le nombre de participants est grand, donc plus l'organisation est complexe et ouverte sur l'environnement, plus la définition de la mission risque d'évoluer rapidement dans les situations de changement majeur. Or, une des réalités incontournables de la mise en œuvre est l'effet du décalage sur la diffusion le long de la ligne hiérarchique. Un changement radical prend du temps à se réaliser et, dans tout changement initié d'en haut, il y a un écart important dans l'évolution d'un dossier selon que l'on se trouve au sommet ou à la base de la pyramide hiérarchique.

Dans le processus de redéfinition de la gestion, la constance dans le temps joue un rôle important pour renforcer la crédibilité de la stratégie. En effet, le décalage menace la cohérence dans l'action et, dans un environnement turbulent, il peut arriver que le changement soit tellement rapide qu'il dépasse les capacités d'absorption de la structure hiérarchique. Un cadre du groupe Exploitation à Hydro-Québec disait à ce sujet :

«...Il y a eu tellement de changements à Hydro que les gens se disent : « Bof, c'est une mode, ça va passer. » Devant les patrons, ils ne disent rien et (ensuite) ils font ce qu'ils veulent. »

Les changements trop fréquents ont un impact négatif sur le processus de redéfinition de la gestion. Ils réduisent la compréhension qu'ont les membres de l'organisation du changement et affaiblissent la mobilisation parce qu'ils remettent en cause l'engagement des dirigeants face au changement. Dans cette situation, le processus de diffusion vertical se désintègre. Les cadres intermédiaires passent leur temps à éteindre les feux et à tenter de maintenir les opérations.

56 Compte tenu de ces tensions entre processus horizontal et vertical de diffusion, leur interaction doit viser à établir un équilibre dynamique. L'objectif ultime étant d'arriver à une configuration où les liens entre les différents groupes et entre les niveaux hiérarchiques sont assez forts pour empêcher la fragmentation de l'entreprise. Toutefois on voit bien qu'il est possible de trouver des situations où le processus horizontal tend à se renforcer au détriment du processus vertical et vice-versa.

Une des caractéristiques du modèle de la diffusion que nous proposons est donc de traiter l'organisation comme une matrice d'interconnexions qui est toujours en fluctuation. Les réseaux d'interaction se reconstruisent continuellement, donc les différents cycles de diffusion sont toujours en interaction. Ce modèle tient compte du fait qu'une organisation complexe n'est pas monolithique et que toutes les parties de l'organisation ne changent pas au même rythme et dans le même sens. Dans les pages qui suivent, nous résumons 
notre modèle et le situons dans le contexte de la littérature en théorie des organisations, en stratégie et en communication. Puis, nous identifions quelques avenues de recherche qui sont suggérées par ce modèle.

\section{Conclusion}

Dans cet article, nous avons proposé un modèle de la diffusion d'un changement radical initié par la haute direction. Nous avons donc porté notre attention sur l'interaction entre la haute direction, qui veut informer les gens et les convaincre de s'impliquer dans la transformation de leur organisation, et les membres de l'entreprise qui interprètent le changement, c'est-à-dire qui y réagissent et agissent en réponse à celui-ci. Autrement dit, nous nous sommes intéressée autant à la partie émergente qu'à la partie délibérée de la diffusion stratégique. Nous avons cherché à mieux comprendre comment se reconstruisait la réalité sociale dans une organisation complexe en transformation.

Nous proposons que le processus de diffusion stratégique, dans une situation de changement radical, peut être conçu comme l'interaction entre un processus de redéfinition de la réalité organisationnelle et un processus de transformation de l'espace organisationnel (Bartunek, 1984 : Ranson \& Hinings, 1980).

D'une part, le processus de redéfinition entraîne une déconstruction (et la reconstruction) de la structure organisationnelle, donc des réseaux qui lient les acteurs entre eux. D'autre part, la nature des nouveaux liens qui sont formés change le sens qui est donné à l'information qui circule dans l'organisation donc redéfinit la réalité organisationnelle, transforme le cadre dominant.

61 Prenant cette conception comme point de départ, nous avons développé un modèle dynamique du processus de diffusion stratégique qui permet d'intégrer redéfinition et restructuration de la réalité organisationnelle. En effet, notre démarche nous amène à tenter de spécifier les mécanismes par lesquels évoluent simultanément le contenu stratégique et l'espace organisationnel.

Dans les organisations stables, c'est-à-dire celles qui évoluent de façon incrémentale, les processus de redéfinition de la mission et de redéfinition de la gestion sont étroitement couplés ce qui les rend difficiles à distinguer, sauf de façon très générale. Une réflexion sur le changement radical permet d'identifier les caractéristiques distinctives de ces deux processus, tant au niveau de l'évolution du contenu que de la structure.

63 Nous avons défini le processus de redéfinition de la mission comme un processus de diffusion médiatisé qui structure latéralement l'organisation. Dans l'esprit de la direction, la redéfinition de la mission est pour l'essentiel terminée au moment de la diffusion, alors que, pour le reste de l'organisation, elle ne fait que commencer. C'est cette tension entre les deux perspectives qui révèle l'aspect émergent du processus de redéfinition de la mission et fait voir son effet structurant sur la réalité organisationnelle.

Le focus de cette discussion étant la définition du territoire organisationnel, l'interaction entre la vision de la direction et celles des groupes résulte en une redéfinition de l'organisation, par exemple, en termes de territoire commun ou partagé, ouvert ou fermé.

65 Le processus de redéfinition de la gestion structure verticalement l'organisation. Alors que, dans le processus de diffusion vertical, les dirigeants mettent l'accent sur la transmission d'information à partir du sommet, les membres de l'organisation le voient 
surtout comme une occasion de réinterpréter la vision pour se l'approprier. Il s'agit non pas de reproduire la vision, mais de la traduire pour qu'elle soit pertinente dans la pratique.

Le rôle que joue l'émergence dans le processus de redéfinition de la gestion dépend de la qualité de la relation entre supérieur et subordonné, particulièrement de l'existence d'une forme de réciprocité à la place de l'utilisation exclusive du pouvoir formel. Si la relation entre supérieur et subordonné ne fait pas de place aux initiatives de la base, les activités émergentes ne seront pas canalisées et pourront devenir dysfonctionnelles.

Le sujet privilégié dans cet échange est la définition des tâches, c'est-à-dire que le discours dans ce processus est, avant tout, axé sur la pratique. C'est principalement par le biais d'une discussion des conséquences de la vision dans l'action que tous les membres de l'organisation participent directement au processus de diffusion stratégique.

Finalement, le processus de diffusion stratégique peut générer trois cycles de diffusion. Le cycle d'exclusion, qui résulte de problèmes au niveau de la redéfinition de la mission, est une boucle de rétroaction qui engendre un accroissement de l'écart entre la vision des dirigeants et celle des groupes exclus. Dans le cycle de désengagement, l'écart entre la vision des dirigeants et la stratégie réalisée est généré par des problèmes au niveau de la redéfinition de la gestion. La troisième boucle, le cycle d'intégration, est caractérisée par une interaction entre redéfinition de la mission et redéfinition de la gestion qui produit un rapprochement entre la stratégie négociée et la stratégie articulée.

Le processus de diffusion stratégique peut donc être conçu comme l'évolution des différentes parties de l'organisation à l'intérieur de ces cycles et entre ces cycles, évolution qui détermine le sens profond et la forme de l'organisation transformée.

Cette perspective permet, entre autres, de concevoir la différentiation horizontale et la différenciation verticale, deux thèmes importants dans la littérature sur la théorie des organisations (Cummings, 1983; Galbraith, 1973 : Lawrence \& Lorsch, 1969 ; Mintzberg, 1979), non pas de façon statique, comme des réponses plus ou moins satisfaisantes à des contingences organisationnelles, mais plutôt de façon dynamique, comme des phénomènes dont il faut comprendre l'émergence et l'évolution.

71 En fait, le changement radical initié par la direction ne fait, dans un premier temps, que remettre en cause le contrat qui existait entre les groupes. C'est dans l'interaction pour accomplir l'activité organisationnelle que les territoires et les tâches se redéfinissent et donnent un sens à la nouvelle réalité et, en même temps, la modifient.

Du point de vue de la littérature en stratégie, cette approche permet de décrire plus précisément les activités qui sont liées aux processus de formulation et de mise en œuvre de la stratégie. Alors qu'ils sont généralement définis en termes de leur résultat, de leur produit, et considérés finalement comme des boîtes noires, cette étude permet d'arriver à un modèle qui décrit leur fonctionnement respectif et l'interaction entre eux.

En ce qui concerne plus spécifiquement la littérature en communication, ce modèle permet de concevoir l'étude des réseaux de communication dans une perspective diachronique, plutôt que statique (Monge \& Eisenberg, 1987). Surtout, elle permet de lier deux problématiques qui sont traitées de façon isolée dans la littérature, celle sur les réseaux et celle sur la création- de sens dans les organisations, et de les intégrer dans une approche qui permet de faire le pont entre les niveaux micro et macro organisationnels (Weick, 1983 ; Eisenberg \& Riley, 1988). 


\section{Les conséquences de cette étude pour la recherche}

74 Cette étude suggère plusieurs avenues de recherche basées sur la prémisse que la diffusion stratégique est, à la fois, un processus horizontal de redéfinition de la mission et un processus vertical de redéfinition de la gestion. Premièrement, l'étude approfondie de plusieurs cas de changement radical initié par la haute direction est nécessaire pour mieux comprendre l'impact de différents types de changement sur le processus de diffusion. Il serait intéressant de varier les types de changement radical, par exemple selon le contexte: changement réactif vs changement anticipatif, selon le contenu: changement défensif vs changement offensif, ou encore selon les résultats : changement réalisé vs changement avorté.

Un tel programme de recherche, nous permettrait de développer et de vérifier l'utilité des éléments constitutifs du modèle: les processus de redéfinition de la mission et de redéfinition de la gestion. Entre autres, ces études de cas pourraient nous permettre de dégager certains patterns d'évolution du contenu et leurs caractéristiques. Par exemple, si l'ouverture du processus de diffusion horizontal à un plus grand nombre de participants entraîne une évolution plus rapide de la vision négociée, en quoi cela affectet-il la complexité de la vision et son degré de cohérence interne?

La deuxième avenue de recherche qui découle de cette étude concerne l'aspect dynamique de notre modèle de diffusion. Ainsi, quels patterns de structuration horizontale et verticale facilitent à la fois l'intégration stratégique et la direction stratégique? Autrement dit, quels patterns de diffusion permettent d'atteindre un certain niveau de consensus (entre les groupes) sans diluer la vision stratégique et un degré d'autonomie sans entraîner la dérive stratégique? Par exemple, le cas de la compagnie Toshiba (Trevor, 1988) suggère que l'intégration de représentants des employés syndiqués et des cadres intermédiaires au processus de diffusion horizontal peut être compatible avec une ligne hiérarchique forte, ce qui n'était pas le cas à HydroQuébec. Quels sont les facteurs liés à une telle évolution du processus?

Finalement, la comparaison de différents types d'organisations complexes pourrait éclairer des aspects intéressants du processus de diffusion stratégique. Par exemple, les différences entre groupes d'activités soulèvent la question suivante : le champ d'activités des sous-groupes a-t-il une influence sur le cycle de diffusion (cycle d'exclusion ou cycle de désengagement), dans lequel ils sont susceptibles de s'engager? Par exemple, Lawrence \& Lorsch (1969) suggèrent que les groupes qui ont à faire face à plus d'incertitude sont plus préoccupés par les questions de contenu, ces groupes seraient-ils alors plus sensibles à la question de représentativité, tandis que les groupes ayant à faire face à une incertitude modérée seraient plus concernés par les questions sociales, donc de redéfinition de la gestion et seraient plus sensibles à la qualité de la relation supérieursubordonné?

En somme, la présentation de ce modèle de diffusion stratégique n'est qu'une première étape qui suggère la pertinence pour comprendre le processus de changement radical de considérer la façon dont les membres de l'organisation le comprennent et le vivent. 


\section{BIBLIOGRAPHIE}

ALLAIRE, Y., Firsirotu, M. E. « How to Implement Radical Strategies in Large Organizations », Sloan Management Review, 26, printemps : 19-34,1985.

ANDREWS, K. The Concept of Corporate Strategy, Dow Jones-Irwin, Homewood, Illinois, 3rd edition, 1987.

ANDERSON, D. « Une démarche pour revitaliser les grandes entreprises », Revue française de gestion, mars-avril-mai 1986.

BARNARD, C. The Functions of the Executive, Harvard University Press, xe edition, 1968.

BARTUNEK, J. « Changing Interpretive Schemes and Organizational Restructuring : the Example of a Religious Order », Administrative Science Quaterly, 29,1984.

BIBEAULT, D. Corporate Turnaround, McGraw-Hill, New York, 1982.

BOURGEOIS, J. \& Brodwin, D. «Strategic Implementation : Five Approaches to an Elusive

Phenomenon », Strategic Management Journal, 5,1984.

BURGELMAN, R. « À Model of the Interaction of Strategic Behavior, Corporate Context and the Concept of Strategy », Academy of Management Review, 8,1983.

Business Week, «Caught in The Middle : Six Managers Speak Out on Corporate Life ». September : $80-88,1988$

CHAFFEE, E. E. « Three Models of Strategy », Academy of Management Review, 10,1985.

CUMMINGS, L. « The Logics of Management », Academy of Management Review, 8,1983.

DAFT, R. \& Huber, G. « How Organizations Learn : A Communication Framework », in Research in The Sociology of Organizations, N. DiTomaso \& S. Bacharach (eds.), JAI Press, vol. 5,1987.

DAFT, R. L. \& Weick, K. E. « Toward a Model of Organizations as Interpretation Systems », Academy of Management Review, 19,1984.

DEMERS, C. « La diffusion stratégique en situation de complexité. Hydro-Québec, un cas de changement radical », thèse de doctorat non publiée, Ecole des HEC, 1990.

EISENBERG, E. \& Riley, P. « organizational Symbols and Sense-Making », in Handbook of Organizational Communication, G. Goldhaber \& G. Barnett (eds.), Ablex, 1988.

FIRSIROTU, M. «Strategic Turnaround as Cultural Revolution : The Case of Canadian National Express ». Unpublished Doctoral Dissertation, McGill University, 1984.

GALBRAITH, J. Designing Complex Organizations, Addison-Wesley, 1973.

HAFSI, T. « Du management au métamanagement : les subtilités du concept de stratégie », Gestion , 10,1985.

HAFSI, T. \& Demers, C. Le changement radical dans les organisations complexes. Le cas d'Hydro-Québec, Gaëtan Morin éditeur, 1989.

HEDBERG, B. « Reframing as a Way to Cope with Organizational Stagnation », International Institute of Management, Berlin, October 1974. 
HEDBERG, B. \& Jönsson, S. « Strategy Formulation as a Discontinuous Process », International Studies of Management \& Organization, 7,2,1977.

HREBENIAK, L. \& Joyce, W. Implementing Strategy, MacMillan Publishing, 1984.

ISABELLA, L. « Evolving Interpretations as a Change Unfolds : How Managers Construe Key Organizational Events », Academy of Management Journal, 33,1991.

KRONE, K., Jablin, F. \& Putnam, L. « Communication Theory and Organizational Communication : Multiple Perspectives », in Handbook of Organizational Communication, F. Jablin et al (eds.), Sage Publications, 1987.

LAWRENCE, P. \& Lorsch, J. Organizations and Environment, R. D. Irwin, 1969.

MC DANIEL JOHNSON, B. Communication: The Process of Organizing, Allyn and Bacon, Toronto, 1977.

MINTZBERG, H. The Structuring of Organizations, Prentice-Hall, 1979.

MINTZBERG, H.\& Waters, J. « Of Strategies, Deliberate and Emergent », Strategic Management Journal, 1985.

MONGE, P. \& Eisenberg, E. « Emergent Communication Networks », in Handbook of Organizational Communication, F. Jablin et al (eds.). Sage Publications, 1987.

MOSS KANTER, R. The Change Masters, Simon Shuster, 1983.

NADLER, D. \& Tushman, M. Organizational Frame Bending : Principles for Managing Reorientation », Academy of Management Executive, 3,1989.

NEILSEN, E. \& Hayagreeva Rao, M. « The Strategy-Legitimacy Nexus : A Thick Description », Academy of Management Review, 12,1987.

NONAKA, I. « Toward Middle-Up-Down Management : Accelerating Information Creation », Sloan Management Review, 9,1988.

PETTIGREW, A. The Awakening Giant Continuity and Change in Imperial Chemical Industries, Basil Blackwell, 1985.

PUTNAM, L. \& Pacanowsky, M. Communication and Organizations, An Interpretive Approach, Sage Publications, 1983.

ROGERS, E. \& Shoemaker, F. Communication of Innovations, Free Press, 1971,2 $2^{\text {nd }}$ edition.

RANSON, S. Hinings, B. \& Greenwood, R. « Tthe Structuring of Organization Structures ", Administrative Science Quaterly, 25,1980.

SELZNICK, P. Leadership in Administration, Row, Peterson and Company, 1957.

SIMON, H. Administrative Behavior, Free Press, 3rd edition, 1976.

TREVOR, M. Toshiba's New British Company, Policy Studies Institute, London, 1988.

TUSHMAN, M. \& Romanelli, E. « Organizational Evolution : A Metamorphosis Model of Convergence and Reorientation », dans L. Cummings \& B.Staw (eds.), Research in Organizational Behavior, JAI Press, 7,1985.

WEICK, K. «Organizational Communication: Toward a Research Agenda », in Communication and Organizations, An Interpretive Approach, L. Putnam \& M. Pacanowsky (eds.), Sage Publications, 1983.

WESTLEY, F. « Middle Managers and Strategy : Microdynamics of Inclusion », Strategic Management Journal, 11,1990. 


\section{NOTES}

1. Le regroupement des cadres en association est particulier aux sociétés d'Etat et aux organismes gouvernementaux où la sécurité d'emploi est garantie. Dans l'entreprise privée, on retrouve essentiellement le même phénomène (voir l'article dans Business Week en référence) de démobilisation, mais il ne se manifeste pas nécessairement de façon tangible.

\section{RÉSUMÉS}

L'objectif de cet article est de proposer un modèle du processus de diffusion stratégique qui tient compte de l'interaction entre l'interprétation des dirigeants et celles des autres membres de l'organisation, dans une situation de changement radical. Le processus de diffusion y est donc conçu non seulement comme un processus de transmission d'information mais également comme un processus interactif de création de sens. La diffusion stratégique y est décrite comme l'interaction entre deux processus qui s'articulent respectivement autour de la redéfinition de la mission et de la redéfinition de la gestion. Le premier de ces processus est une dynamique horizontale: une négociation entre les différentes parties de l'organisation, tandis que le deuxième est une dynamique verticale : une chaîne d'échanges entre supérieur et subordonné.

This article proposes a model of strategic diffusion process, taking into account both the vision of top management and the interpretation of other organization's members in the context of a radical change. The diffusion process is thus conceived both as an information transmission process and an interactive sense-making process. Strategic diffusion is described as resulting from the interaction of two processes: the redefinition of the mission and the redefinition of management philosophy. The first of these processes restructures the organization horizontally : it is a negociation between the different units of the organization. The second restructures the organization vertically : it is a chain of exchanges between superior and subordinate.

\section{AUTEUR}

\section{CHRISTIANE DEMERS}

Christiane Demers est professeur adjoint à l'École des Hautes Études Commerciales de Montréal où elle enseigne la stratégie d'entreprise. Ses intérêts de recherche sont le changement dans les organisations et la communication organisationnelle. Ses publications récentes incluent Le changement radical dans les organisations complexes. Le cas d'Hydro-Québec (avec Taieb Hafsi), Gaetan Morin éditeur, 1989 et « Redressement et transformation : une comparaison du point de vue de la diffusion ", dans les Actes du 1 er Colloque International en gestion stratégique. Publications Economica à paraître. 